Gynäk. Rdsch. 1983;23(suppl. 2):I-IV

\title{
Contents, Vol. 23, Supplement 2, 1983
}

\section{Foreword 1}

Editorial

Epidemiology and Symptomatology of Trichomoniasis

Rüttgers, $\mathrm{H} \quad 3$

Trichomoniasis: Mechanism of Infection, Recurrences and

Reinfections in Man

Bedoya,J.M 10

Diagnostic Problems

Jenny, J.W 16

Conventional Therapy of Trichomoniasis

Richter, R 23

Vaccination with SolcoTrichovac. Immunological Aspects of a

New Approach for Therapy and Prophylaxis of Trichomoniasis

in Women

Pavic, R.; Stojkovic, L

Serological Study with SolcoTrichovac, a Vaccine against

Trichomonas vaginalis Infection in Women

Milovanovic, R.; Grcic, R.; Stojkovic, L

IgA Antibodies in the Vaginal Secretion after Vaccination with

SolcoTrichovac

Milovanovic, R.; Grcic, R.; Stojkovic, L 46

Changes in the Vaginal Flora of Trichomoniasis Patients after

Vaccination with SolcoTrichovac

Milovanovic, R.; Grcic, R.; Stojkovic, L 50

Effects of Vaccination with SolcoTrichovac on the Vaginal Flora and

the Morphology of the Doederlein Bacilli

Goisis, M.; Magliano, E.; Goisis, F 56

Contents IV

Clinical Experience Using SolcoTrichovac in the Treatment of

Trichomonas Infections in Women

Lorenz, U.; Rüttgers, $\mathrm{H}$

64

SolcoTrichovac in the Prophylaxis of Trichomonad Reinfection.

A Randomized Double-Blind Study

Litschgi, M 72

SolcoTrichovac in Medical Practice. An Open, Multicentre Study to

Investigate the Antitrichomonal Vaccine SolcoTrichovac

Rippmann, E.T 
The Therapeutic and Prophylactic Efficacy of SolcoTrichovac in Women with Trichomoniasis. Investigations in Cairo

Elokda, H.H.; Andrial, M

85

Author Index 89

Subject Index 90 